\title{
Reducing the Training Times of Neural Classifiers with Dataset Condensing
}

\author{
Se-Ho Choi and Peter Rockett \\ Department of Electronic and Electrical Engineering, \\ University of Sheffield \\ Mappin Street, Sheffield, S1 3JD, UK \\ \{shc,pir@bartok.shef.ac.uk\}
}

\begin{abstract}
In this paper we apply a $k$-nearest-neighbour-based data condensing algorithm to the training sets of multi-layer perceptron neural networks. By removing the overlapping data and retaining only training exemplars adjacent to the decision boundary we are able to significantly speed the network training time while achieving an undegraded misclassification rate compared to a network trained on the unedited training set. We report results on a range of synthetic and real datasets which indicate that a speed-up of an order of magnitude in the network training time is typical.
\end{abstract}

Keywords: Neural networks, data editing, pattern classifiers

\section{Introduction}

Neural networks have been shown to be a valuable non-parametric pattern classification technique which - subject to certain conditions - can approximate the posterior probability of class membership [1]. Due to their computational compactness during recall, multilayer perceptrons (MLPs) have attracted much attention and it is this neural architecture we consider here. The principal drawback of MLPs is that their training is approximately $O\left(N^{3}\right)$ and thus scales unattractively. This has lead to a great deal of research aimed at reducing the size of the training set of examples to a set which nonetheless captures the critical information about the classification mapping in hand.

Kraaijveld \& Duin [2] applied the well-known multiedit algorithm to the training set of a neural network which, since it removes class overlap in the dataset, resulted in faster training and an unambiguous stopping criterion for the training. Along with many others, Kraaijveld \& Duin noted that only the subset of exemplars adjacent to the decision boundary is required to train a classifier but their application of a condensing algorithm produced poor results because the very small amount of data remaining after condensing was clearly insufficient to adequately constrain the decision surface of the subsequently trained MLP.

A number of other authors have attempted to pre-select training exemplars for neural networks with the objective of reducing the training time while not degrading classification performance compared to a network trained conventionally on the whole set 
of prototype patterns. Hara \& Nakayama [3] employed a data pairing method where they selected the pairs of exemplars drawn from different classes but with the minimum Euclidean separation although the sparseness of the edited set produced means it will probably be quite sensitive to sampling effects. Cachin [4] compared a number of techniques which use different strategies to make more frequent presentations to the partially trained network of those patterns for which the training error is highest. Although the number of presentations of the training set required to attain a given error rate is reduced by maybe half compared to conventional cycling through the dataset, a single presentation in the technique studied by Cachin comprises multiple repeats of individual patterns within the training set and it is unclear whether there is an overall saving in $C P U$ time. Further, it is also unclear whether increasing the relative frequency of presentation of some patterns in the training set implicitly changes the priors on the problem, thus violating one of the conditions for the network to learn the posterior probability [1]. Leisch et al [5] have used an active pattern selection strategy to significantly reduce the computational burden of cross-validation of MLPs while Hwang et al [6] have employed query-based selection of training points to finetune an almost-trained network.

In the present work we have employed a condensing algorithm which uses nearestneighbour estimates of the posterior probability to significantly reduce the size of the training set and therefore the training time. In doing this we have taken pains not to over-edit the training set such that the reduced dataset is unable to constrain the decision boundary sufficiently or lacks resilience to sample size effects; we thus address the principal shortcoming of the condensing procedure presented in [2]. In the next section we describe our method and show results on a range of synthetic and real data in Section 3. The results are discussed in Section 4 and conclusions offered in Section 5.

\section{The Data Condensing Algorithm}

By definition, any datum for which the posterior probability is 0.5 lies on the decision surface whereas data with posterior probabilities of either 0 or 1 are remote from the decision surface and therefore of minor importance to proper training. Considering (without loss of generality) a two-class problem: Our aim is to include in the condensed training set only those data for which $0.5 \leq P(C \mid X) \leq 0.5+\lambda$ where $P($. $)$ is the posterior probability, $C$ is the class, $X$ is the input vector and $\lambda$ controls the degree of data reduction. We thus eliminate data which overlap the other class(es) resulting in improved training [2]. In essence we select two bands or 'strips' of non-overlapping data either side of the decision surface to guard against excessive sensitivity to sampling. In this way we can constrain the location of the decision boundary without permitting the network to perform spurious extrapolations in the pattern space.

To estimate the posterior probabilities we have used the $k$-nearest-neighbour $(k$ $N N$ ). If a datum satisfies the bounds on its probability given above, it is copied into the condensed dataset. The condensing algorithm employed here is thus particularly simple and straightforward to implement.. 


\section{Results}

In this work we have considered only MLPs with sigmoidal transfer functions and used conventional error back propagation minimising a squared error metric to train the MLPs. We have applied the present data condensing method to a range of synthetic and real problems.

\subsection{One-Dimensional Overlapping Gaussians}

The 1D Gaussian represents probably the simplest class of problem but is nonetheless instructive. We generated 500 data randomly drawn from two overlapping Gaussian distributions, $N(1,1)$ and $N(3,1)$. Assuming equal priors, the decision boundary is where the variate equals 2.0 and 158 of the data overlap the other class implying a misclassification of about $16 \%$. We have trained an MLP with one input node, two hidden nodes and one output layer on this problem and the error rates and the computational effort and are shown in Table 1. (For convenience we define the 'computational effort' of training to be the product of the number of iterations to convergence and the size of the training set normalised to the corresponding product for the unedited dataset.) The first two rows of Table 1 show the variation of the size of the training set with the parameter, $\lambda$.

The error rate for each of the trained MLPs was estimated using a validation set of 4000 data independent of the training set. Both the misclassification rate and the computational effort have been averaged over fifty independently initialized networks. We have (approximately) optimized the learning rate and momentum parameters used in the backpropagation algorithm; we believe this to be a reasonable comparison since although we have used fixed learning parameters, in practice these would typically be determined using an optimising line search technique.

In considering the results in Table 1, the reference figures for networks trained on the original, unedited dataset of 1000 members are : Error rate of $16.07 \%$ and a (normalized) computational effort of unity. (Throughout this paper we gauge the validation error from the minimum figure attained with reference to the independent validation set.) Two things are immediately apparent from Table 1: Firstly, the misclassification rate is not statistically different between the network trained on the original dataset and the networks trained on the condensed datasets. This is gratifying since this meets one of our original objectives. Second, the training effort is reduced by up to an order of magnitude by data condensing but all other things being equal, one would expect the dataset for $\lambda=0.05$ (41 members) to result in faster training than, say the dataset for $\lambda=0.45$ (612 members). Given that we are defining computational effort to be the product of the number of iterations to convergence and the cardinality of the training set, clearly the dataset for $\lambda=0.05$ is much slower to converge.

Hara \& Nakayama [3] have considered this second phenomenon in relation to their data pairing technique and suggest that initially, the (randomly assigned) decision boundary is far from its correct location. If all the training data are grouped in a narrow region either side of the true decision boundary, the derivatives which drive the backpropagation algorithm will be exceedingly small since we are evaluating these at 
the data points. (Equivalently, all of the neurons will be operating in the saturation regions of the sigmoid transfer characteristic which are almost horizontal.) Thus the rate at which the network adjusts to the true decision boundary will be slow. Datasets constructed from larger values of $\lambda$ have wider 'bands' of data around the decision surface thus ameliorating the problem of small derivatives. The computational effort results in Table 1 show a minimum for $\lambda=0.25$ which is consistent with this explanation.

To speed convergence still further we have taken the condensed dataset of 41 data for $\lambda=0.05$ and added to it 40 randomly selected data - twenty from each class - with posterior probabilities in the range of $0.55 \leq P(C \mid X) \leq 1.0$. Thus if the initial random decision boundary is far from the true boundary the randomly selected data scattered throughout the space should ensure that the derivatives of the error function do not vanish. The comparable averaged figures for the $\lambda=0.05+$ randomly selected data are : Misclassification rate $=15.92 \%$ and a computational effort of 0.02 , a fifty-fold reduction compared to training with the unedited dataset. This represents something of a paradox since adding more data results in faster training.

\subsection{Two-Dimensional Synthetic Problem}

We have applied the present technique to a $2 \mathrm{D}$ two-class classification problem shown in Fig 2(a). We have used 2000 points from each class as a training set (which included 289 overlapping data) and we estimated the posterior probabilities over 100 nearest-neighbours $(k=100)$. The original dataset is shown in Figure 1(a) and the condensed dataset for $\lambda=0.05$ is shown in Figure 1(b). Various condensed datasets were used to train a 2-8-1 MLP and again results are presented for 'optimal' learning parameters in Table 2; the validation results were obtained over an independentlygenerated dataset of 14,486 members.

The first column of Table 2 shows the results for training with the original unedited dataset; as before, the error and computational effort results have been averaged over fifty independent trainings. The trends in the results are identical to the $1 \mathrm{D}$ problem presented previously and similarly, we have also trained with a dataset formed by the union of the condensed set for $\lambda=0.2+214$ random selected points for which the posterior probability was $>0.7$. In this case, the error rate was $6.35 \%$ and the computational effort was 0.07 , in line with the previous findings.

\subsection{Real Datasets: Breast Cancer \& Character Recognition}

We have employed the current method on two real datasets: a breast cancer dataset and a handwritten character recognition dataset both from the UCI Database [7]. The breast cancer data comprised 683 members (we have excluded 16 data with missing attributes) and this was randomly divided into a training set of 483 and a test set of 
200. The training set comprised 167 benign examples and 316 malignant; the test set comprised 73 benign and 127 malignant. The data was condensed using a $k$-value of 30 and we have trained 9-9-1 MLPs. Table 5 shows the results.

From the handwritten character dataset [7] we have selected the "B" and "D" data to form a two-class problem. We have selected a training set of 1268 members (630 "B" and 638 "D") and a test set of 303 (136 "B" and 167 "D"). Using a $k$-value of 60 we have trained a 16-10-1 MLP and the results are shown in Table 6.

In summary, both the real and synthetic datasets display consistent results: the use of condensed datasets speeds training significantly whilst not degrading the classification performance.

\section{Discussion}
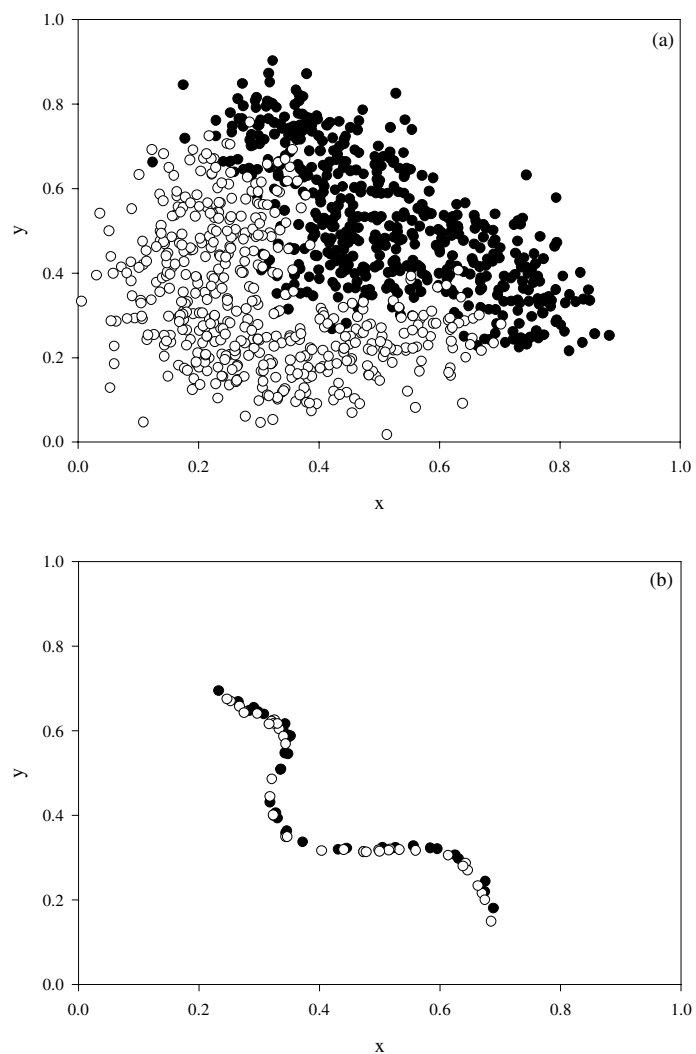

Figure 1: 2D Synthetic dataset. (a) shows the original dataset and (b) shows the condensed dataset for $\lambda=0.05$

One of the pre-conditions under which a neural network learns to approximate the posterior probability on a classification problem is that the training sample reasonably reflects the data distribution on the underlying or parent problem. At a point in the region of overlapped data, the MSE training criterion ensures the network output is the best compromise between competing forces by approximating the posterior probability. If the overlapping data are removed from a training set then clearly the network can no longer hope to approximate the posterior probability and such a trained network's capabilities as a classifier need to be carefully re-evaluated. Formally, Bayesian decision theory involves two stages : Firstly, the evaluation of the posterior probability for each class; second, picking the class with the largest posterior 
probability $[$ Traditionally, neural networks have been used to estimate the posterior probability and the second stage has been performed implicitly. If we remove class overlap we would expect the network to produce a step change in output on passing through the decision surface. In this context, it is clear that the trained network has learned the Bayesian decision mapping in its entirety rather than just the input-spaceto-posterior-probability mapping which it would do if trained on the unedited dataset. We thus conclude that networks trained on condensed datasets still implement maximum a posteriori (MAP) classifiers but dispense with the (implicit) decision stage. To illustrate this, network outputs for the 1D Gaussian problem (Section 3.1) are shown in Fig 2. The network trained on the unedited dataset - Figure 2 - approximates the posterior probability whereas the network trained with the condensed dataset approximates a binary decision.

By employing suitably edited training sets we have demonstrated here that savings in the MLP training time of factors of 4-50 are possible compared to training with the unedited training set although the exact speedup will depend on the mapping, MLP architecture, etc. In practice we need to compare the overall training times (time to perform the data editing plus the reduced time to train the network). While the data condensing algorithm presented here is $O\left(N^{2}\right)$ where $N$ is the size of the dataset, the backpropagation algorithm is approximately $0\left(N^{3}\right)$. Thus, as $N$ becomes larger, we would expect the condensing-preceding-training approach to yield favourable reductions in overall computation time. In practice, however, this asymptotic argument understates the potential time savings : Since there is no principled method to determine in advance the optimum number of hidden units for an MLP for a given problem, it is normal practice to train a range of networks to find the one with the fewest hidden neurons that is able to satisfactorily learn the problem (i.e. fastest recall). The condensing algorithm clearly needs to be run only once for given problem so in practice the (more favourable) time comparison should be the time to perform $M$ networktrainings on the unedited dataset versus the time for one run of the condensing algorithm plus $M$ network trainings on the condensed dataset. Taken together with the effective elimination of overfitting, we believe the present approach represents a significant advance in the training of neural network classifiers. To give a concrete timing result, we have trained a 16-16-1 MLP both on an original training set of 16000 items constructed from the character recognition data [7] as well as the condensed set of 1500 data. The total training times are as follows: Original dataset $=16.296 \mathrm{~min}-$ utes; Condensed dataset $=6.137$ minutes (comprising 3.943 minutes for the data condensing algorithm and 2.194 minutes for the MLP training) indicating that the present method achieves significant time savings even on moderately sized datasets.

\footnotetext{
${ }^{1}$ Pedantically, the decision stage assigns the class with the minimum cost or risk. Here, without loss of generality we are assuming both types of error have equal costs.
} 


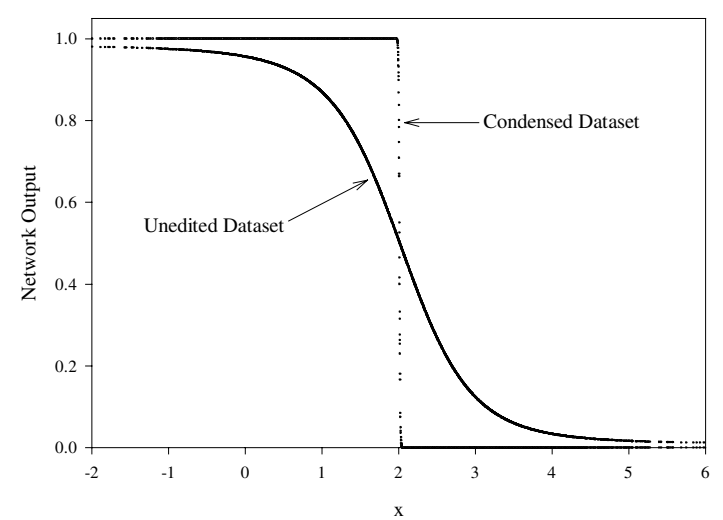

Figure 2: Comparison of network outputs for MLPs trained with the original 1D Gaussian dataset and condensed dataset. The network outputs for class ' $\mathrm{B}$ ' - which is the complement of that for class ' $A$ ' - have been omitted for clarity

\section{Conclusions}

In this paper we have reported the results of training multi-layer perceptrons (MLPs) with datasets condensed by a technique which uses $k$-nearest neighbour estimates of posterior probability. In essence, we select two non-overlapping 'strips' of data either side of the decision boundary such that $0.5 \leq P(C \mid X) \leq 0.5+\lambda \quad$ where $P(C \mid X)$ is the conditional

probability of class membership and $\lambda$ is a parameter which controls the degree of data reduction. We have demonstrated that the MLPs so trained have misclassification rates not statistically different from networks trained on the original unedited dataset but the computational effort needed is significantly reduced. It is difficult to generalise about the savings in MLP training time since this will depend on the problem in hand but for the representative datasets considered here, an order of magnitude is typical.

We have been able to further speed the initial stages of training by adding a small percentage of randomly selected data to the training set which ensures that the gradient values driving the backpropagation algorithm do not become unacceptably small when the initial, randomly-assigned decision boundary is far from the true decision surface.

\section{Acknowledgments}

The breast cancer data used here was obtained originally from Dr. William H. Wolberg of the University of Wisconsin Hospitals, Madison whom we gratefully acknowledge.

\section{References}

[1]Richard M D, Lippmann R P, "Neural Network Classifiers Estimate Bayesian a Posterior Probabilities", Neural Computation, Vol 3, pp 461-483, 1991.

[2] Kraaijveld M A, Duin R P W, “An Optimal Stopping Criterion for Backpropagation Learning”, Neural Network World, Vol 1, No 6, pp 365-370, June 1991.

[3] Hara K, Nakayama K, "Training Data Selection Method for Generalization by Multilayer Neural Networks”, IEICE Trans Fundamentals, vol E81-A, No 3, pp 374-381, 1998. 
[4]Cachin C, "Pedagogical Pattern Selection Strategies", Neural Networks, Vol 7, No 1, pp 175-181, 1994

[5]Leisch F, Jain L C, Hornik K, "Cross-Validation with Active Pattern Selection for NeuralNetwork Classifiers," IEEE Trans on Neural Networks, vol 9, No 1, pp 35-41, 1998.

[6] Hwang J-N, Choi J J, Oh S, Marks R J, "Query-Based Learning Applied to Partially Trained Multilayer Perceptrons", IEEE Trans Neural Networks, Vol 2, No 1, pp 131-136, 1991

[7] Downloadable from tp://ftp.ics.uci.edu/pub/learning-databases/

Table 1 : Misclassification \& computational effort results for various training sets for true 1D Gaussian problem

\begin{tabular}{|c|c|c|c|c|c|c|c|c|}
\hline$\lambda$ & 0.05 & 0.1 & 0.15 & 0.2 & 0.25 & 0.3 & 0.35 & 0.45 \\
\hline No of Data & 41 & 85 & 107 & 159 & 209 & 268 & 354 & 612 \\
\hline Error [\%] & 16.07 & - & 15.92 & - & 15.92 & - & 15.95 & 16.05 \\
\hline Computation & 0.70 & - & 0.13 & - & 0.09 & - & 0.12 & 0.1 \\
\hline
\end{tabular}

Table 2 : Misclassification \& computational effort results for various training sets for the 2D synthetic problem

\begin{tabular}{|c|c|c|c|}
\hline$\lambda$ & N/A & 0.2 & 0.3 \\
\hline No of Data & 4000 & 278 & 492 \\
\hline Error [\%] & 6.37 & 6.4 & 6.34 \\
\hline Computation & 1 & 0.19 & 0.09 \\
\hline
\end{tabular}

Table 3 : Misclassification and computational effort for various training sets for the breast cancer data. The first column shows the results for the unedited dataset and the last column the results for $\lambda=0.3+26$ random data

\begin{tabular}{|c|c|c|c|c|}
\hline$\lambda$ & N/A & 0.2 & 0.3 & $\begin{array}{c}0.3+\text { random } \\
\text { data }\end{array}$ \\
\hline No. of data & 483 & 78 & 104 & 104 \\
\hline Error [\%] & 3.5 & 3.0 & 3.5 & 3.5 \\
\hline Computation & 1 & 0.09 & 0.12 & 0.04 \\
\hline
\end{tabular}

Table 4 : Misclassification and computational effort for various training sets for the handwritten character data. The first column shows the results for the unedited dataset and the last column the results for $\lambda=0.2+129$ random data

\begin{tabular}{|c|c|c|c|c|}
\hline$\lambda$ & N/A & 0.2 & 0.3 & $\begin{array}{c}0.2+\text { random } \\
\text { data }\end{array}$ \\
\hline No of data & 1268 & 323 & 452 & 452 \\
\hline Error [\%] & 3.3 & 2.97 & 3.3 & 3.3 \\
\hline Computation & 1 & 0.39 & 0.41 & 0.19 \\
\hline
\end{tabular}

